\title{
Música contemporánea y estrategias de aprendizaje de una nueva pieza musical en la clase de piano
}

\author{
Michel André Cara Jara \\ Email: michel.cara@gmail.com
}

\begin{abstract}
Resumen: El presente trabajo de investigación, se centra en el estudio del proceso de aprendizaje a corto plazo de una nueva pieza de música contemporánea en una clase de piano funcional en la Facultad de Artes de la Universidad de Chile. Los participantes $(n=10)$ leen 4 veces la pieza seleccionada y tienen la posibilidad de practicar dos minutos entre cada interpretación. Se analizan los datos de la performance, así como la implicación de la maestra y sus estudiantes en la generación de estrategias dinámicas de aprendizaje. Los resultados sugieren la existencia de proto-perfiles de aprendizaje.

Palabras-clave: Aprendizaje a corto plazo; estrategias dinámicas de aprendizaje; performance musical; música contemporánea, clase de piano.

\section{Música contemporânea e estratégias de aprendizagem de uma nova peça musical na aula de piano}

Resumo: Este trabalho de pesquisa concentra-se no estudo do processo de aprendizagem, em curto prazo, de uma nova peça de música contemporânea em uma aula de piano funcional na Faculdade de Artes da Universidade do Chile. Os participantes $(n=10)$ liam a peça selecionada por 4 vezes com a possibilidade de praticar por dois minutos entre cada interpretação. Foram analisados os dados sobre a performance e o envolvimento do professor e os seus alunos na geração de estratégias dinâmicas de aprendizagem. Os resultados sugerem a existência de perfis de protoaprendizagem.

Parabras-chave: Aprendizagem de curto prazo; estratégias dinâmicas de aprendizagem; performance musical; música contemporânea; aula de piano.

\section{Contemporary music and learning strategies for a new musical piece in piano classes}

\begin{abstract}
This research work studied the short-term learning process of a new piece of contemporary music in a functional piano class at the Faculty of Arts of the University of Chile. The participants $(n=10)$ read the selected piece 4 times and were allowed to practice for two minutes between each performance. The performance data are analyzed as well as the involvement of the teacher and students in the generation of dynamic learning strategies. The results suggest the existence of proto-learning profiles.
\end{abstract}

Keywords: Short-term learning; dynamic learning strategies; musical performance; contemporary music, piano classroom.

\section{Introducción}

Nos hemos interesado en analizar el proceso de aprendizaje de una nueva pieza de música contemporánea en estudiantes de piano funcional que son alumnos de una misma profesora. A través de una metodología inspirada en trabajos anteriores (LEHMANN, ERICSSON, 1996; DRAKE, PALMER, 2000; ROSEMANN, ALTENMÜLLER, FAHLE, 2015) nos proponemos responder a las siguientes preguntas: ¿Cómo opera el proceso de aprendizaje en interacción? ¿Qué tipos de estrategias utilizan los estudiantes? ¿Cuál es el efecto de una pequeña fase de práctica en el aprendizaje de una nueva pieza de música? ¿Cómo evolucionan dichas estrategias a través de las fases de práctica? ${ }^{1}$ 


\subsection{Aprendizaje y competencias musicales}

La adquisición de competencias musicales puede tener lugar a distintos intervalos (i.e., corto y largo plazo) y depender de varios factores que no son fácilmente influenciables por condiciones externas ${ }^{2}$ (GRUHN, RAUSCHER, 2008). En efecto, el desarrollo de estrategias de procesamiento, toma y recuperación de la información musical estaría regulado, desde un punto de vista pedagógico, por una fase de retroalimentación que permite a su vez que se modifiquen dichas estrategias y por lo tanto los recursos cognitivos (Autor, 2016). Según Drake y Palmer (2000), la búsqueda de la fluidez es un indicador clave de la adquisición de competencias musicales a corto plazo. Desde esta perspectiva, la adquisición de competencias consistiría en "construir, recuperar y asociar conocimientos que permitan la comprensión de la situación, la planificación y el control de la acción” (MARTENIUCK, 1986; RICHARD, 1998, citados en KERMARREC, 2004, p. 17).

Según Anderson (1995), el conocimiento puede ser presentado en formatos procedimentales o declarativos, lo que implica que la metodología de enseñanza determina la calidad del aprendizaje. Las estrategias procedimentales serían más eficaces que las declarativas, y a pesar que el comportamiento observado sea similar, éstas comprometen diferentes tipos de activaciones cerebrales (ALTENMÜLLER et al., 2000). En efecto, el aprendizaje intensivo, induce un incremento a corto plazo en la actividad cerebral. A la inversa, el aprendizaje a largo plazo, produce una disminución en dicha actividad y sería facilitado por estrategias procedimentales. Esto implica un cambio de las activaciones desde las áreas prefrontales del cerebro hacia aquellas que se ocupan de tareas específicas. Este fenómeno se conoce como desplazamiento anterior-posterior (GRUHN, RAUSCHER, 2008). Por esta razón es importante comprender cómo estos mecanismos se regulan durante el aprendizaje. Se ha observado que durante el aumento del rendimiento en tareas específicas ${ }^{3}$, las activaciones cerebrales implicadas disminuyen (véase Gruhn, 2004). El efecto a largo plazo del aprendizaje depende entonces de diferentes patrones de activación. Estas evidencias sugieren según el autor, por un lado, que el aumento de la activación cerebral a corto plazo está vinculada principalmente a procesos de memorización y, en segundo lugar, que la internalización subconsciente, en ciertos tipos de aprendizaje, compromete programas motores automatizados que afectan estructuras cerebrales más profundas. Las adquisiciones a corto plazo requieren entonces la integración de nuevos conocimientos para que persistan a largo plazo.

Una competencia que es determinante en la música occidental es la lectura a primera vista, definida como la capacidad de leer una partitura por la primera vez sin el beneficio de la práctica (WOLF, 1976). Leer a primera vista requiere por parte del intérprete un dominio tanto de la técnica como del repertorio, por lo tanto, implica la movilización de conocimientos previos (mecanismos top-down) y de recursos cognitivos específicos que pueden estar determinados según el tipo de repertorio, como es el caso de la memoria espacial a corto plazo en la música contemporánea (Autor, 2017).

\subsection{Cuestionarios y protocolos verbales como herramienta de información sobre el proceso de aprendizaje}

Cuestionarios y protocolos verbales (véase ERICSSON, SIMON, 1980) pueden ser utilizados durante o después de la práctica musical lo que permitiría analizar los diferentes tipos de estrategias que utilizan los músicos. A diferencia de los protocolos verbales, los cuestionarios permiten obtener diferentes tipos de información sin afectar la ejecución de 
la tarea. Presentaremos brevemente algunas investigaciones en el dominio musical que han utilizando la metodología del protocolo verbal, las que se relacionan con el análisis de las estrategias de aprendizaje (MCPHERSON, MCCORMICK, 2006), el papel de los mecanismos de atención (CHAFFIN et al., 2003) y el tipo de representaciones involucradas en el trabajo de memorización de una nueva pieza de música (WILLIAMON, VALENTINE, VALENTINE, 2002).

McPherson y McCormick (2006) muestran que la habilidad en la performance de músicos jóvenes en etapa de formación, se correlaciona con los niveles de autoeficacia. Los autores solicitan a un grupo de pianistas que evalúe, en una escala de 10 a $100 \%$, cuan confiados se sienten de tocar correctamente en un examen de grado. Las respuestas (en porcentajes) se introducen en un modelo que consta de varios niveles: eficacia personal, estrategias cognitivas, regulación de la práctica, práctica formal y práctica informal. Finalmente, la autoeficacia resulta ser la variable que mejor predice el éxito de los músicos en las pruebas de fin de ciclo.

Los mecanismos de atención también han sido abordados por medio de técnicas de protocolos verbales y cuestionarios aplicados a los músicos. Chaffin et al. (2003) estudian, en un pianista experimentado, cómo opera el intercambio de recursos atencionales entre la imagen global de la pieza y los aspectos técnicos, durante la preparación del tercer movimiento del Concerto Italiano de J.S. Bach. Los autores muestran, al analizar los protocolos verbales, que antes de la fase de preparación de la pieza, se establece una jerarquía de acción ${ }^{4}$ al reconocer la imagen artística de la pieza. De esta manera es posible, gracias a los protocolos verbales, diferenciar las acciones más intuitivas de aquellas más deliberadas. La imagen artística avanza a medida que avanza la práctica y se elabora junto a conceptos técnicos, la estructura musical, la memoria y la interpretación. La capacidad de un experto para anticipar acontecimientos en vista de una futura interpretación (CHASE, 1983; GLASER, CHI, 1988; GOBET, SIMON, 1996; CHAFFIN et al., 2003) le permiten al intérprete tomar mejores decisiones especialmente en el momento de la práctica o la preparación de la pieza.

Por su parte, Williamon et al. (2002) realizan un estudio sobre las estrategias de memorización de dos expertos pianistas con más de 18 años de experiencia al estudiar una pieza de J.S. Bach (i.e., Polonesa en sol menor, del libro de Anna Magdalena) ${ }^{5}$ bajo ciertas condiciones experimentales. En la primera condición, los pianistas deben imaginar que tocan la pieza y tienen que comentar sobre cada aspecto de la pieza. En la segunda condición, los pianistas tocan la pieza de memoria, indicando oralmente los aspectos en los que se centran. Las categorías de análisis comprenden representaciones visuales, kinestésicas y auditivas. Los análisis de las verbalizaciones muestran que en la primera condición los pianistas tienen una visión general de la pieza y al mismo tiempo se concentran en ciertos elementos salientes de la partitura. El procesamiento de los aspectos globales y locales estaría generalmente relacionado con la estructura de la música. En cuanto a la segunda condición, los comentarios también se centran en los diferentes niveles de la estructura musical, sin embargo, los aspectos visuales son más recurrentes que en el caso de la primera condición. El autor interpreta estos resultados citando a Clarke (1988), para quien los comentarios sobre la estructura generativa de la pieza serían exteriorizados durante la interpretación de la obra. Por otra parte, las estrategias de memorización dependen de la alternancia de segmentos largos y cortos durante el estudio. Esto permitiría a los músicos desarrollar estructuras de recuperación de la información, necesarias para el procesamiento de estructuras musicales. La implementación de este tipo de estrategias depende del nivel de experticia de los músicos (KINTSCH, VAN DIJK, 1978; DRAI-ZERBIB, BACCINO, 2005). 
De esta manera la estructura formal de la música actúa como un sistema jerárquico en el que se organiza el foco atencional.

\subsection{Problematización}

Si bien las metodologías de resolución de problemas (véase DEWEY, 1910 1916, véase también BRUNER, 1961) pueden ayudar al estudiante a aumentar su capacidad para resolver problemas y a tener una cierta autonomía en su aprendizaje, podrían ser cuestionadas por las siguientes razones: (1) la forma de su inclusión en el proceso; (2) la frecuencia de uso; (3) su validez en términos pedagógicos. Según Sweller (1988), la adquisición de esquemas (relacionados con el comportamiento experto) podría oponerse a la utilización de una metodología de resolución de problemas. Los autores concluyen que los mecanismos necesarios para la resolución de problemas y la adquisición de esquemas son diferentes. Desde esta perspectiva, la adquisición de la experticia podría retrasarse si nos basamos únicamente en este tipo de metodología. Respecto al tipo de estrategias utilizadas para aprender una nueva canción, Nielsen (1999) muestra que el desarrollo de habilidades metacognitivas es otro beneficio para la solución de problemas relacionados con la interpretación y la técnica. Sin embargo, estas habilidades sin conocimiento previo serían de poca utilidad, lo que pone de relieve la importancia de un programa de trabajo que considere ambos aspectos.

\subsection{Objetivos del estudio}

Se espera que los estudiantes movilicen distintos tipos de estrategias a lo largo de las diferentes fases de práctica en la fase de práctica y logren estabilizar las variables de la performance (i.e., tempo, timing y errores) en las fases iniciales del aprendizaje. Desde un punto de vista pedagógico, esperamos que la introducción de una nueva metodología de evaluación del aprendizaje a corto plazo, sea relevante para fomentar la interacción dentro del aula, el desarrollo de estrategias dinámicas de aprendizaje, la construcción de los conocimientos de los estudiantes, así como el desarrollo de meta-competencias disciplinarias.

\section{Metodología}

El presente estudio se basa en información cuantitativa (i.e., datos de la performance) y cualitativa (i.e., cuestionarios y protocolos verbales), en la forma de un estudio de caso y a través de un "análisis interpretativo" que permite la segmentación, codificación y categorización de los datos (PHELPS, FERRARA, GOOLSBY, 1993; MARCZYK, DEMATTEO, FESTINGER, 2005; FIREMAN, 2007). Los datos de la performance se analizan a partir de archivos MIDI.

\subsection{Participantes}

Participan 10 estudiantes de Licenciatura en Música de la Universidad de Chile que cursan la asignatura de piano funcional (entre el tercer y el quinto año). Los participantes tienen entre 21 y 26 años de edad (media $=23.4, D S=1.5$ ) su experiencia con práctica del piano varía entre 3 y 11 años (media $=6.05, D S=2.89$ ), tienen entre 2 y 8 años de experiencia en lectura musical (media $=4.8, D S=2.29$ ), y practican entre 60 a 180 minutos diariamente (media $=88.1, D S=39.02$ ). 


\subsection{Material musical}

“Algunas preguntas” de Juan Amenábar es una pieza atonal breve que fue encargada por la Facultad de Artes de la Universidad de Chile y publicada en una antología por la misma Facultad (ALARCÓN, 1993). La obra, que tiene un carácter pedagógico presenta una estructura en forma de mosaico, con tres secciones características: primera sección, compases 1 a 6; segunda sección compases 7 a 11 y tercera sección, compases 12 a 17. Se destaca un aumento de las pulsaciones en la segunda sección, como la presencia de motivos de tensión y de resolución (ver figura 1). La división en secciones responde a problemas de organización visual a lo largo de ejes de expansión y contracción de la información en lugar de una lógica de estructura formal. Por lo tanto, esta división en secciones permite realizar análisis estadísticos (efecto de aprendizaje) relacionados con las variables de la performance.

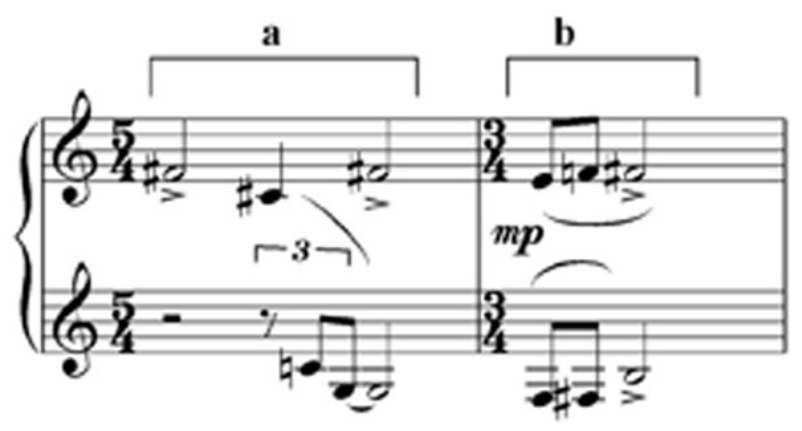

\subsection{Equipamiento musical}

Los músicos tocan en un teclado Roland HP335 conectado a una interfaz de M-Box Digidesign que envía los datos a ordenador portátil. Para la grabación de datos en formato MIDI, utilizamos el software CUBASE SX4. El músico recibe un retorno audio a través de los altavoces del teclado.

\subsection{Criterios de análisis y de evaluación}

La evaluación de la performance y de los aspectos cualitativos se abordan según los criterios presentados en la siguiente tabla.

Tabla 1 - Resumen de los aspectos a considerar durante la evaluación del proceso de aprendizaje a corto plazo

Evaluar Con la finalidad de:

Las estrategias implementadas por los participantes Identificar los mecanismos cognitivos implicados (WILLIAMON et al., 2002; LEHMANN, ERICSSON, 1996) y contrastarlos con la metodología de evaluación de la profesora

Los mecanismos implicados en el aprendizaje en Estudiar cómo los estudiantes se representan interacción su propia forma de aprender. Estudiar el tipo de codificación y la modalidad de recuperación de la información musical (visual, auditiva o kinestésica).

El saber-hacer en la performance 


\subsection{Cuestionarios y protocolos verbales}

Se elaboran cuestionarios y protocolos verbales para los estudiantes. El análisis de unidades de sentido nos permite construir categorías que puedan traducir objetivamente el concepto de aprendizaje en interacción y, al mismo tiempo, den cuenta de las representaciones subjetivas que los estudiantes construyen de su propio aprendizaje. La idea es obtener detalles sobre la relación maestro-alumno y cómo se realiza la transmisión de los conocimientos musicales (i.e., cómo influyó la profesora en su aprendizaje) de manera de poder entender cómo el alumno adhiere a las enseñanzas de la maestra (i.e., en qué medida su profesora lo marcó).

\subsection{Procedimiento}

La prueba de lectura consiste en leer cuatro veces la pieza "Algunas preguntas" con la posibilidad de dos minutos de entrenamiento entre cada interpretación. Las mediciones de la performance se registraron con la grabación de datos en formato MIDI y con registro audiovisual. En la segunda parte del experimento, se entrega un cuestionario a los estudiantes, se realizan los protocolos verbales y se realiza una entrevista a la profesora para obtener información sobre la metodología de trabajo de su clase.

\section{Resultados}

Para cada variable dependiente, se realiza un análisis de la varianza de medidas repetidas ANOVA con dos factores intra-sujetos (2: sección, ensayo) y experticia como factor entre sujetos. Como hemos dicho antes, la pieza fue divida en tres secciones. Un participante fue eliminado del cálculo del timing por presencia de outliers con más de dos desviaciones estándar de la media en más de una sección.

\subsection{Performance}

Los resultados de las variables provenientes de la performance se muestran en las Tablas 2, 3 y 4. Se calculó el nivel de experticia de los pianistas sustrayendo el número medio de errores al valor medio del tempo. La anterior fórmula ha sido utilizada en estudios precedentes realizados con pianistas profesionales (Autor, 2017). De diez pianistas, seis estaban en el quinto año de la Carrera de Licenciatura en Música. Cinco de ellos fueron clasificados como de nivel de los expertos. Otros estudiantes de tercero y cuarto, pertenecen al panel inferior. Todos los músicos del panel de expertos tienen más de cinco años de experiencia en el piano.

Tabla 2 - Estadísticas descriptivas de las variables provenientes de la performance: errores (adiciones, substituciones y omisiones), tempo y timina a lo largo de las 4 interpretaciones de la pieza

\begin{tabular}{lccccc}
\hline & Media & DS & LI & LS & Rango \\
\hline Adiciones & 1.16 & 1.42 & 0.91 & 1.42 & $0-6$ \\
Substituciones & 1.27 & 2.08 & 0.90 & 1.65 & $0-14$ \\
Omisiones & 0.55 & 1.42 & 0.29 & 0.81 & $0-11$ \\
Tempo & 34.98 & 10.13 & 8.78 & 11.33 & $17-63.24$ \\
Timing & 18.96 & 6.94 & 6.12 & 80.1 & $8.58-40.88$ \\
\hline
\end{tabular}

Nota. LI = 95\% intervalo de confianza (límite inferior); LS = 95\% intervalo de confianza (límite superior); 
Las comparaciones planificadas indican que, a partir del segundo ensayo, los participantes tienden a tocar más rápido en la primera sección en comparación con las otras secciones $\left(F(1,8)=19.07, p=.002, \eta_{\mathrm{p}}{ }^{2}=.70 ; F(1,8)=22.09, p=0.002, \eta_{\mathrm{p}}{ }^{2}=.73\right.$; $F(1,8)=25.60, p<.001, \eta_{\mathrm{p}}{ }^{2}=.76$, ensayos 2,3 y 4 respectivamente). Sin embargo, aunque en el primer ensayo dicha diferencia no es significativa $\left(F(1,8)=0.17, p=.69, \eta_{\mathrm{p}}{ }^{2}=.021\right)$ los pianistas tienden a tocar más rápido la tercera sección, lo que sugiere un doble efecto, por una parte, negativo, ya que los pianistas aumentan el tempo sin que sea indicado en la partitura y por otro lado, positivo, ya que se infiere que los participantes han integrado parte de la estructura de la obra (la tercera sección tiene una estructura similar a la primera sección).

A lo largo de los ensayos, las variaciones de tempo (timing) aumentan globalmente, lo que sugiere la implementación de mecanismos expresivos e interpretativos de la pieza. Sin embargo, esto se hace en detrimento de una ejecución rigurosa en la primera sección. Por su parte, el aumento significativo del tempo durante los ensayos indica un claro efecto del aprendizaje de la obra.

Tabla 3 - Valores de F, probabilidades y eta cuadrado parcial de los análisis de la varianza ANOVA conducidos por cada variable estudiada: tempo, timing y errores (i.e., adiciones, sustituciones y omisiones)

\begin{tabular}{lllllll}
\hline Variables & $\mathrm{n}$ & \multicolumn{2}{l}{ Sección } & $\eta_{\mathrm{p}}{ }^{2}$ & Ensayo & $\eta_{\mathrm{p}}{ }^{2}$ \\
\hline Adiciones & 10 & $10.72 * * *$ & .54 & $8.1{ }^{* * *}$ & .47 \\
Substituciones & 10 & 2.13 & $n s$ & .19 & $5.97 * *$ & .40 \\
Omisiones & 10 & 2.45 & $n s$ & .21 & $2.88(\mathrm{p}=.54)$ & .24 \\
Tempo & 10 & $13.40 * * *$ & .59 & $13.99 * * *$ & .61 \\
Timing & 9 & $37.65 * * *$ & .82 & $7.38 * * *$ & .48 \\
\hline
\end{tabular}

Timing $\frac{9}{* p<.05 ; * * p<.005 ; * * * p<.001}$

Tabla 4 - Analysis post-hoc (Tukey HSD). Valores medios y probabilidades de nivel de los factores sección (Sec) y ensayo (Ens). En paréntesis las desviaciones estándar

\begin{tabular}{lccccccc}
\hline Variables & Sec. 1 & Sec. 2 & Sec. 3 & Ens. 1 & Ens. 2 & Ens. 3 & Ens. 4 \\
\hline Adiciones & $0.72 \mathrm{a}$ & $1.9 \mathrm{~b}$ & $0.88 \mathrm{a}$ & $2.03 \mathrm{a}$ & $1.23 \mathrm{ab}$ & $0.93 \mathrm{~b}$ & $0.47 \mathrm{~b}$ \\
& $(1.11)$ & $(1.88)$ & $(1.24)$ & $(1.47)$ & $(1.63)$ & $(1.20)$ & $(0.82)$ \\
Substituciones & 0.83 & 1.31 & 1.67 & $2.43 \mathrm{~b}$ & $1.06 \mathrm{a}$ & $0.73 \mathrm{a}$ & $0.86 \mathrm{a}$ \\
& $(1.48)$ & $(1.49)$ & $(2.90)$ & $(3.06)$ & $(1.78)$ & $(1.14)$ & $(1.43)$ \\
Omisiones & 1.88 & 1.04 & 0.43 & 1.09 & 0.43 & 0.43 & 0.24 \\
& $(0.50)$ & $(1.88)$ & $(1.39)$ & $(2.45)$ & $(0.86)$ & $(0.82)$ & $(0.62)$ \\
Tempo & $37.99 \mathrm{a}$ & $33.2 \mathrm{~b}$ & $33.75 \mathrm{~b}$ & $28.34 \mathrm{a}$ & $33.7 \mathrm{ab}$ & $37.31 \mathrm{bc}$ & $40.58 \mathrm{c}$ \\
& $(10.43)$ & $(9.75)$ & $(8.98)$ & $(6.72)$ & $(8.98)$ & $(8.88)$ & $(10.56)$ \\
Timing & $24.48 \mathrm{~b}$ & $16.06 \mathrm{~b}$ & $16.33 \mathrm{~b}$ & $15.45 \mathrm{a}$ & $18.53 \mathrm{ab}$ & $20.27 \mathrm{~b}$ & $21.57 \mathrm{~b}$ \\
& $(7.38)$ & $(4.91)$ & $(4.64)$ & $(4.66)$ & $(6.91)$ & $(6.56)$ & $(8.0)$ \\
\hline
\end{tabular}

Nota. Las letras (grupos Tukey ANOVA) indican cuales son los valores medios que se diferencian entre los diferentes grupos (a o b) o bien aquellos que comparten posición con otros grupos (ab o bc). ${ }^{*} p<.05 ;{ }^{* *} p<.01$.

\subsection{Experticia}

Se observa un efecto principal de la experticia (i.e., capacidad de interpretar la pieza con la mayor velocidad posible y cometiendo el menor número de errores) sobre las omisiones de notas $\left(F(1,8)=5.3 p=, 050, \eta_{\mathrm{p}}{ }^{2}=.40\right)$, las adiciones $(F(1,8)=40.00, p$ $\left.<.001, \eta_{\mathrm{p}}{ }^{2}=.83\right)$, pero no sobre las substituciones $\left(F(1,8)=2.21 p=.18, \eta_{\mathrm{p}}{ }^{2}=.22\right)$. Se 
observa, por otra parte, una interacción estadísticamente significativa experticia-ensayo en las omisiones de notas $\left(F(3,24)=4.72, \mathrm{p}=.009, \eta_{\mathrm{p}}{ }^{2}=.37\right)$. Las comparaciones planificadas muestran que los músicos con mayor experiencia cometen menos omisiones solamente en el primer ensayo $\left(F(1,8)=6,12, \mathrm{p}=0,038, \eta_{\mathrm{p}}{ }^{2}=.40\right)$. Respecto a la variación de tempo (timing), una interacción estadísticamente significativa experticia/sección, muestra que los pianistas con mayor experiencia logran estabilizar el tempo entre secciones $(F(2,14)=7,65$, $p<0.005, \eta_{\mathrm{p}}{ }^{2}=.52$ ). Las comparaciones planificadas muestran que dichas diferencias conciernen únicamente a la primera sección $\left(F(1,7)=10,79, p=.013, \eta_{\mathrm{p}}{ }^{2}=.54\right)$.

Estos resultados sugieren que los pianistas más expertos son capaces de interpretar integralmente la partitura con menos variaciones de tempo entre secciones y respetando el tempo señalado por el compositor. Por otra parte, los pianistas menos avanzados tienden a sustituir notas durante la lectura a primera vista de la pieza y tienen más dificultad para mantener un tempo constante, lo que sugiere que tienen cierta dificultad para elegir un tempo que se ajuste a sus posibilidades. Lo anterior se refleja particularmente en la primera sección donde se observan diferencias según el grupo de nivel. Cabe señalar que, si bien los grupos experimentales son pequeños, y por tal razón es necesario tomar con cautela estos resultados, el tamaño del efecto es frecuentemente de moderado a alto.

Se aborda paralelamente la relación entre los conceptos de base evaluados por la maestra ${ }^{6}$ y las variables de la performance. La variable experticia correlaciona con positivamente con los años de experiencia previa en el piano $(r(8)=0.73, p<0.01)$ como a su vez con los conceptos de base intencionados por la profesora $(r(8)=0.64, p<0.05)$.

\subsection{Cuestionarios y verbalizaciones}

Para abordar la relación entre dichos conceptos de base y la metodología implementada por la profesora, hemos formulado a los estudiantes las siguientes preguntas: ¿Cómo influyó la profesora en su aprendizaje? ¿En qué medida su profesora lo marcó? Por su parte para abordar el proceso de aprendizaje y las interacciones dentro de la clase de piano, hemos preguntado a los estudiantes las siguientes preguntas: ¿Cómo trabajó la pieza? ¿Cómo se dio cuenta de su progreso? En un primer momento, para analizar los datos de cuestionarios y verbalizaciones, hemos identificado diferentes unidades de sentido y categorías elaboradas a partir de ciertos conceptos significativos. Una parte importante de las respuestas se refiere al comportamiento heurístico-adaptativo expresado en unidades de sentido relacionadas con la "confianza". El análisis fue realizado considerando cada indicador por separado (ver Tabla 5). Se decidió integrar categorías suplementarias en el análisis con el fin de observar las representaciones visuales, auditivas o kinestésicas movilizadas por los estudiantes en la codificación y recuperación de información. 
Tabla 5 - Ejemplo de unidades de sentido y de sus categorías asociadas enunciadas en los cuestionarios

\begin{tabular}{|c|c|}
\hline Categorías & Ejemples \\
\hline Heurística & $\begin{array}{l}\text { En un primer momento traté de leer verticalmente nota por nota sin tener un buen } \\
\text { resultado }\end{array}$ \\
\hline Metódica & En un primer momento solfeé los ritmos y luego miré las alteraciones \\
\hline Técnica & $\begin{array}{l}\text { Pulsación lenta...Observar la tesitura (registro) y, desde esos elementos, encontrar la } \\
\text { posición de las manos }\end{array}$ \\
\hline Confianza & $\begin{array}{l}\text { Según la confianza que yo adquiría, cada vez pude tocar un poco más rápido y sin } \\
\text { cometer errores }\end{array}$ \\
\hline Global & $\begin{array}{l}\text { En un primer momento leí la pieza del inicio al final con el objetivo de tener una } \\
\text { noción de las dificultades. }\end{array}$ \\
\hline Local & A lo largo de la experiencia me focalicé en los saltos de voz... \\
\hline Auditiva & ...al tocarlas juntas (las manos) con el fin de escuchar como ellas debían sonar juntas \\
\hline Visual & Creo que no me di cuenta de algunas articulaciones \\
\hline Kinestésica & $\begin{array}{l}\text { A partir de la tercera lectura aproximadamente, había incorporado los movimientos } \\
\text { que debía realizar con las manos }\end{array}$ \\
\hline Interpretativa & $\begin{array}{l}\text { Noté nuevos errores y al final de la experiencia me pude consagrar a la expresividad } \\
\text { apoyándome en la misma lectura. }\end{array}$ \\
\hline
\end{tabular}

\subsubsection{Representatividad intra-preguntas}

El análisis de la representatividad intra-preguntas, enfatiza la relación entre los diferentes indicadores al interior de cada pregunta (ver tabla 6). Es posible que el tipo de pregunta influya en el porcentaje de representatividad de los indicadores ${ }^{7}$ (SEBILLOTTE, 1991), o simplemente que algunas preguntas no sean relevantes. A pesar de dichas limitaciones, el análisis nos permite verificar la representatividad de los indicadores en cada pregunta, compararlas para analizarlas en mayor detalle (sin sobrestimar su representatividad) y de esta manera sacar algunas conclusiones sobre la interacción profesor-alumno.

Se observa una variabilidad importante de los indicadores en relación a los diferentes ítems (ver tabla 6). Las diferentes preguntas parecen ser relevantes para evaluar los indicadores relacionados con la técnica, la confianza, lo kinestésico y la interpretación, que, en efecto, resultan mejor representados. A excepción de las respuestas sobre la metodología de trabajo de la pieza (ver tabla 6, línea 1), los indicadores relacionados con la técnica y la confianza parecen estar representados en la misma proporción y son los indicadores que mejor se adecuan al tipo de pregunta. Observamos que los últimos cinco indicadores (ver tabla 6, columnas 7-11) proporcionan poca información sobre los ítems metacognición, influencia de la profesora y aprendizaje significativo a excepción de los indicadores heurística y metacognición. En efecto, los tres últimos ítems (ver tabla 6, líneas 3-5) se refieren a las representaciones subjetivas que los estudiantes construyen sobre su propia forma de aprender y no serían pertinentes para la evaluación de ciertos indicadores. El primer ítem (trabajo pieza) se refiere más bien a aspectos procedimentales. 
Tabla 6 - Porcentaje de representatividad intra-pregunta. Cómo trabajó usted la pieza (Trabajo pieza). Cómo se dio cuenta de su progreso (Metacognición), De qué manera la profesora influyó en su aprendizaje (Influencia profesora), en qué medida su profesora lo marcó (Ap. Significativo)

\begin{tabular}{llllllllllll}
\hline Ítems & Obs & Tec & Conf & Kin & Int & Heur & Met & Glob & Loc & Vis & Aud \\
\hline Trabajo pieza & 31 & 29 & 6.5 & 3.2 & -- & 16.1 & 16.1 & 12.9 & 12.9 & 3.2 & -- \\
Metacognición & 18 & 44.4 & 27.7 & 11.1 & 5.5 & 5.5 & -- & -- & -- & -- & -- \\
Influencia profesora & 14 & 57.1 & 21.4 & 7.1 & 14.9 & -- & -- & -- & -- & -- & -- \\
Ap. significativo & 16 & 31.3 & 25 & 12.5 & 18.8 & -- & -- & -- & -- & -- & 12.5 \\
Total & 79 & 30 & 14 & 6 & 6 & 6 & 5 & 4 & 4 & 2 & 2 \\
\hline
\end{tabular}

Nota. Número de observaciones (Obs) y porcentaje de cada indicador: técnica (tec), confianza (Conf), heurística (Heur), metódica (Met), visual (Vis), Kinestésica (Kin), Auditiva (Aud), globales (Glob), locales (Loc), interpretación (Int).

\subsection{Práctica entre ensayos}

El análisis de las fases de práctica se realizó a partir de datos audiovisuales. En primer momento, la idea es determinar si los conceptos de base intencionados por la profesora (i.e., respiración, relajación y posición), se manifiestan en la performance de los pianistas, especialmente durante las fases de práctica. Los índices de dichos conceptos de base observados en las grabaciones de video corresponden a comportamientos explícitos que finalmente son categorizados. En un segundo momento, obtendremos más información sobre las diferentes modalidades de práctica: cómo éstas evolucionan a lo largo de los ensayos y si están organizadas de manera jerárquica. Los diferentes comportamientos observados durante la fase de práctica se organizaron en once indicadores de acuerdo con dos criterios: (1) efecto en la continuidad de la lectura y (2) representatividad en las tres fases de la práctica. De acuerdo con estos criterios, se eliminaron tres indicadores de los análisis porque sólo estaban presentes en la segunda práctica (i.e., inicio de la práctica desde la segunda frase musical y la repetición insistente de una frase o de un pasaje con el objeto de verificar). Los demás indicadores se presentan en la Tabla 7.

Un ANOVA de medidas repetidas muestra que existen diferencias significativas entre las fases práctica $\left(F(2,22)=4.33, p<0.05, \eta_{\mathrm{p}}{ }^{2}=.17\right)$ con menos representación de las distintas categorías en la tercera fase de práctica (LSD <0,05). Con el fin de agrupar los indicadores significativos en dos categorías, se calculó una mediana $(\mathrm{m}=11)$. El análisis de las categorías mayormente representativas (ver Tabla 7, columna izquierda) muestra que ciertas categorías pueden ser agrupadas de acuerdo a la frecuencia de observación entre las fases de práctica (ver Figura 2).

Tabla 7 - Número total de observaciones para cada categoría. A la izquierda las categorías que presentan un mayor porcentaje de representación y a la derecha las menos representativas

\begin{tabular}{|c|c|c|c|}
\hline \multicolumn{2}{|l|}{ Indicadores principales } & \multicolumn{2}{|l|}{ Indicadores secundarios } \\
\hline Repetición por verificación & 59 & Reiteración después de un error & 11 \\
\hline Repetición por un error & 54 & Canto de la melodía & 8 \\
\hline Repetición por pérdida de fluidez* & 51 & Mano derecha sola & 7 \\
\hline Lectura desde el inicio & 31 & Práctica mental** & 3 \\
\hline Repetición semi-frase después de un error & 16 & Repetición por verificación & 1 \\
\hline Mano izquierda sola & 13 & --- & \\
\hline
\end{tabular}

Nota. ${ }^{*}$ Hemos observados interrupciones en la lectura que hemos atribuido a una imposibilidad de procesar simultáneamente toda la información musical (aumento de la carga cognitiva). ${ }^{* *}$ Definida por Coffman (1990) como la repetición imaginaria de un procedimiento sin movimiento muscular o sonido. 
Figura 2. Perfiles de evolución de los principales indicadores de las fases de práctica

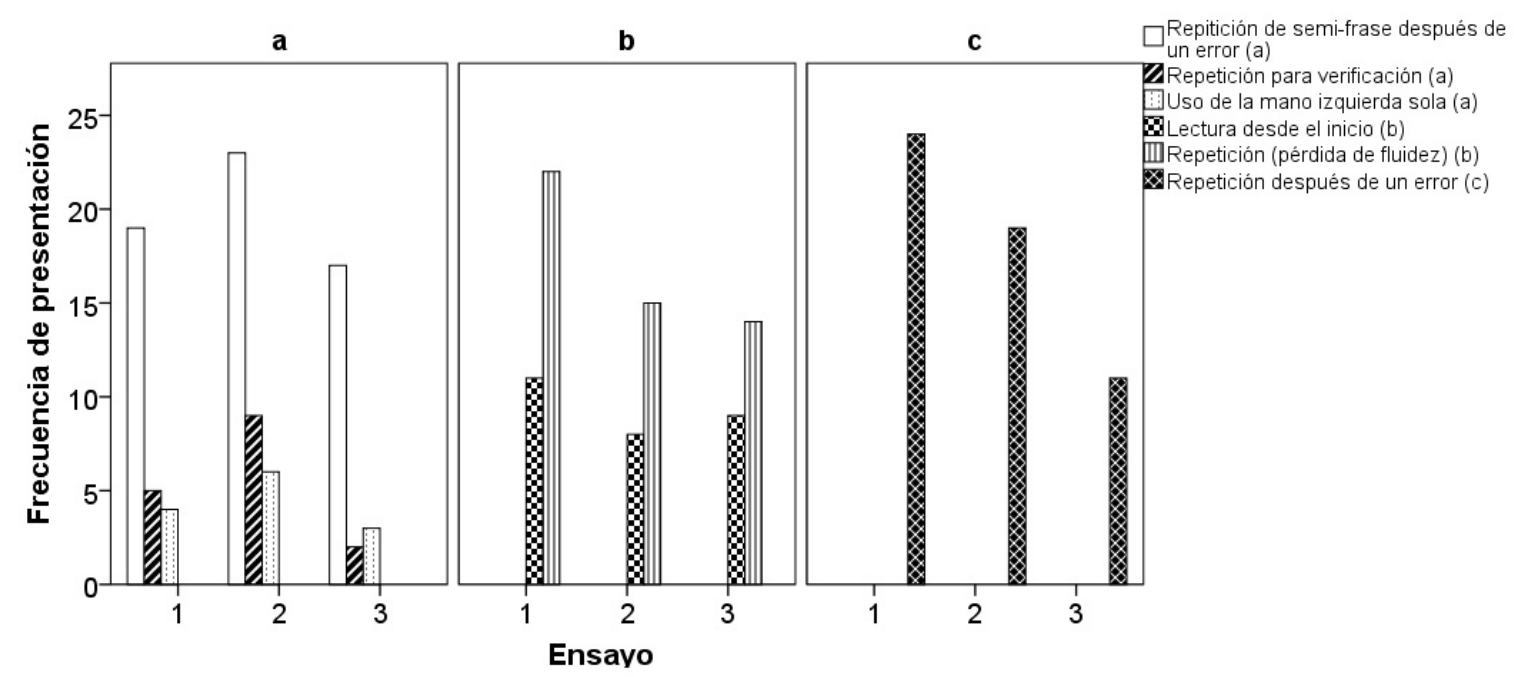

Legenda: En general, hay tres tipos diferentes de comportamiento relacionados con: (a) la integración de la información (b); con la segmentación y (c) con las dificultades en el procesamiento de la información

Se observa una tendencia (no significativa), en los pianistas que cometen más errores, a tocar más lentamente y repetir a nivel local (i.e., en el mismo compás o pulsación) después de un accidente de lectura, lo que se considera un error por pérdida de fluidez $(F(1,8)$ $=3.59, p=0.09, \eta_{\mathrm{p}}^{2}=.18$ ). Del mismo modo, se encontraron correlaciones tendenciales entre el número de errores y el indicador de repetición después de un error $(r(8)=0,6, p$ $=0.06)$.

\section{Discusión}

Hemos estudiado el aprendizaje a corto plazo al interior de una clase de piano utilizando un enfoque integrado. Tomamos en consideración las interacciones dentro de la clase y las concepciones metodológicas de los actores. Las cuatro interpretaciones de la misma pieza y las tres fases de la práctica tuvieron como objetivo estimular la problematización ${ }^{8} \mathrm{y}$ observar la evolución de las performances a lo largo del proceso de aprendizaje. Lo anterior nos permitió estudiar, desde diversos focos, el fenómeno del aprendizaje a corto plazo de una nueva pieza de música contemporánea.

\subsection{Observaciones microgenéticas}

Algunos indicadores, que describen el proceso de aprendizaje (e.g., repetición por pérdida de fluidez) tienen características comunes y en general disminuye su representatividad a medida que avanzan las fases de práctica. Dichos indicadores se construyeron sobre la base de un criterio de continuidad en la lectura, que es según Drake y Palmer (2000) uno de los principales indicadores de la adquisición de competencias musicales. Sin embargo, algunos de ellos relacionados con la integración de la información durante la lectura aumentan en la segunda fase de la práctica. Según los resultados de las observaciones de las fases de la práctica, los pianistas parecen privilegiar las estrategias de integración de la información, de toma de conciencia y de comprensión. A su vez, los pianistas que cometen más errores tienden a repetir y a comprobar las notas después de haber cometido un error lo que dificulta la continuidad del discurso musical. Aunque es difícil establecer la causalidad con respecto a la relación entre error/estrategia utilizada ${ }^{9}$, se 
observan sin embargo comportamientos que apuntan a integrar mejor la información, por ejemplo, a través de la segmentación de estructuras musicales.

$\mathrm{Al}$ observar la representatividad intra-preguntas, los resultados sugieren que la técnica tiene un papel importante en la percepción del estudiante sobre la maestra y sus enseñanzas. Del mismo modo, la confianza se expresa particularmente en los procesos metacognitivos, y presenta menos distancia de los aspectos técnicos cuando se refiere a la significatividad de las enseñanzas. Esta consolidación del aprendizaje tiene lugar a través de la relación maestro-alumno. La diferencia principal está en la forma en que los pianistas trabajan la pieza. El indicador de confianza cede, por un parte, el lugar a los indicadores técnicos y los comportamientos heurísticos o metódicos y, por otro lado, los aspectos interpretativos no aparecen bien representados probablemente por el tipo de pregunta utilizada (ver nota $\mathrm{n}^{0} 7$ ). Estos resultados también sugieren que la profesora influye en el desarrollo de procesos metacognitivos de los alumnos, ya que se espera que la profesora les haya transmitido el conocimiento técnico y la confianza ${ }^{10}$. Ellos también destacan la importancia de la gestión de objetivos personales (BOUFFARD et al., 2001), reconociendo al mismo tiempo que la conciencia del progreso se focaliza en el cumplimiento de ciertos aspectos técnicos e interpretativos obtenidos gracias a la confianza necesaria para llevar a cabo la tarea.

\subsection{Problematización}

Este estudio introduce a partir de la problematización, una metodología de evaluación que resultó ser innovadora en el contexto estudiado. Los pianistas deben estudiar, de manera autónoma una partitura e ir mejorando el rendimiento a lo largo de las cuatro interpretaciones. Esto nos permitió estudiar las estrategias implementadas por los pianistas. Observamos que el proceso de aprendizaje podría enriquecerse mediante la problematización. En nuestra opinión, la lectura a primera vista siempre estará sujeta a la problematización porque el repertorio (en este caso pianístico) es de gran envergadura, sobre todo en el caso de la música contemporánea. Esto es evidente, por ejemplo, en la elección del repertorio lo que según Fireman (2007), estaría asociado a una metodología heurística de resolución de problemas. Por otra parte, el modelo de evaluación que hemos propuesto parece ser compatible con las observaciones de Ausubel (1962) realizadas en el marco del aprendizaje verbal significativo, para quien este tipo de técnica no debe utilizarse para la transmisión directa de un contenido, sino más bien para la evaluación de resultados de aprendizaje "significativo", de la sensibilidad a las fuentes de conocimiento, así como del aprendizaje destinado a la resolución de problemas (p. 116).

En cuanto a la observación de la fase de práctica, hemos constatado la implementación de ciertas estrategias. Por ejemplo, la organización del tiempo disponible para la práctica entre los ensayos, conlleva a una planificación que parece estar sujeta a una jerarquía de acción, tal como lo ha destacado Chaffin et al. (2003) para quienes la elaboración de una imagen artística de la pieza determina la cadena de planificación. Estrategias tales como la repetición después de un error durante la fase de práctica, si son interpretadas como una forma de integrar la información, dan cuenta igualmente de planes de acción implementados por los pianistas. Desde nuestro análisis, las repeticiones conscientes destinadas a la integración de la información corresponden a nuestro indicador "repetición por verificación" (i.e., repetición que no está necesariamente ligada a un error). Por otra parte, el hecho de que el indicador "repetición después de un error" resulte mejor representado que el indicador "repetición semi-frase después de un error" puede indicar 
que la integración de la información se encuentra aún en un nivel local. En efecto, hemos observado que los pianistas que cometen más errores tienden a repetir a nivel local (misma pulsación) o la semi-frase asociada con el error. Esto es consistente con Williamon et al. (2002) quienes muestran que los pianistas, mientas expertos, tienden a utilizar segmentos de práctica más largos y a alternarlos con segmentos más cortos a medida que la práctica avanza (e.g., repetición de la semi-frase después de un error y aumento de la longitud de las secciones de verificación a lo largo de los ensayos). La práctica está así determinada por relaciones jerárquicas que capturan el foco atencional de los pianistas durante la práctica. Lo anterior es posible comprobarlo a partir de las verbalizaciones de los estudiantes. En efecto, tal como lo replantea Sebillotte (1991), la experticia estaría ligada a que los individuos tengan una "representación jerárquica" de la tarea. Por otra parte, las diferentes dimensiones de la problematización implican, más allá del éxito de la tarea, representaciones funcionales y la transformación de las concepciones técnicas que representan los saberes (LE BAS, 2001).

\section{Conclusión}

La presente investigación nos ha permitido aumentar nuestros conocimientos sobre el aprendizaje a corto plazo de una nueva pieza de música contemporánea y sus posibles implicaciones metodológicas. Hemos debido confrontar la problemática de la validez estadística versus la significatividad de los enunciados/verbalizaciones (cuestionarios y protocolos verbales). A pesar de la polarización cuantitativa / cualitativa que podría inducir según Ambert et al. (1995) la implementación de un proceso heurístico, hemos demostrado que estos dos enfoques son compatibles. Por lo demás, se benefician mutuamente ya que el aprendizaje de la lectura es un proceso complejo que debe abordarse de manera integrada e interdisciplinaria de manera de poder comprender mejor los principios del funcionamiento cognitivo que subyacen a la performance (SCHÖN, AKIVA-KABIRI, VECCHI, 2008). Esto también es congruente con gran parte de la investigación en educación que favorece la no polarización de la investigación en el campo educativo (ERCIKAN Y ROTH 2006). Los resultados de este estudio confirman la relevancia de nuestra fórmula para evaluar el nivel de experiencia de los pianistas (tempo-errores). Sin embargo, creemos que esta fórmula no podría extenderse a otros instrumentos sin una previa demostración experimental. En fin, hemos constatado que nuestro enfoque investigativo podría enriquecer los intercambios entre el estudiante, la profesora y el experimentador, lo que se ha manifestado a través de los comentarios positivos de la profesora sobre la utilidad de la implementación de tal sistema evaluativo.

\section{Notas}

1 Ciertos datos del presente trabajo fueron previamente presentados en la conferencia regional de la International Society for Music Education (ISME), septiembre 3-6, Santiago, Chile, 2013.

2 Es particularmente el caso del aprendizaje procedural implícito, regulado por estructuras límbicas (GRUHN, RAUSCHER, 2008).

3 Aprender a diferenciar un modo Dorio y un modo Eolio/Identificar diferentes tipos de acordes.

4 Ver Carver \& Scheier (1998), ver también Rosenbaum, (1987).

5 Como era costumbre en la época como manera de aprender música, ella copió un gran número de manuscritos de su marido. Estas copias hicieron posible recuperar la música de una gran parte de los manuscritos perdidos de J. S. Bach.

6 Evaluación de la profesora que concierne la respiración, la relajación y la posición.

7 Según Sebillotte (1991), la experticia estaría ligada a que los individuos tengan una "representación jerárquica" de la tarea de "lo más abstracta a lo más detallado." Por esta razón, las preguntas formuladas sobre la base de un "cómo" (nivel más bajo en una jerarquía de abstracción) o un "por qué” (nivel de orden superior) pueden conducir a 
respuestas sesgadas (ver GRAESSER, 1981). Sin embargo, permiten “identificar qué acciones corresponden a metas y qué acciones son los medios para alcanzar dichas metas” (p.199).

8 Adherimos a la visión de Marchand (2009) para quien la problematización no tiene necesariamente la finalidad de alcanzar un aprendizaje preciso sino más bien de problematizar una situación musical.

9 Para identificar estrategias globales hemos considerado el error en un sentido amplio.

10 Ver Grangeat, (2010), ver también Flavell (2000), en lo que respecta el nexo entre la teoría del espíritu y la metacognición.

\section{Referencias}

ALARCÓN, M. E. Pequeña antología del compositor chileno para piano. Santiago: Instituto Interamericano de Educación Musical, Facultad de Artes, Universidad de Chile, 1993. 55p.

ALTENMÜLLER, E. et al. The impact of music education on brain networks: evidence from EEG studies. International Journal for Music Education, v. 35, p. 47-53, 2000. https://doi. org/10.1177/025576140003500115

AMBERT, A.-M. et al. Understanding and evaluating qualitative research. Journal of Marriage and the Family, v. 57, n. 4, p. 879-893, 1995.

ANDERSON, J.R. Learning and memory. New York: J. Wiley and Sons, 1995. 448p

AUSUBEL, D. P. Learning by discovery. Educational Leadership, v. 20, n. 2, p. 113-117, 1962.

BOUFFARD, L. et al. La gestion des buts personnels, un apprentissage significatif pour des étudiants universitaires. Revue des Sciences de l'Education, v. 27, p. 503-522, 2001.

BRUNER, J. The act of discovery. Harvard Educational Review, v. 31, p. 21-32, 1961.

DEWEY, J. How we think. Boston: Heath, 1910. 250p.

. Method in science teaching. Science Education, v. 1, n.1, p. 3-9, 1916.

DRAI-ZERBIB, V. ; BACCINO, T. L'expertise dans la lecture musicale: Intégration intermodale. L'Année psychologique, v. 105, p. 387-422, 2005.

DRAKE, C.; PALMER, C. Skill acquisition in music performance: relations between planning and temporal control. Cognition, v. 72, n. 1, p. 1-33, 2000.

ERCIKAN, K.; ROTH, W. M. What good is polarising research into qualitative and quantitative? Educational Researcher, v. 35, n. 5, p. 14-23, 2006. https://doi.org/10.3102/0013189X035005014

ERICSSON, K.; SIMON, H. Verbal reports as data. Psychological Review, v. 87, n. 3, p. 215-251, 1980. http://dx.doi.org/10.1037/0033-295X.87.3.215

CARVER, C. S.; SCHEIER, M. F. On the self-regulation of behavior. Cambridge: Cambridge University Press, 1998. 460p.

CHAFFIN, R. et al. "Seeing the big picture": Piano practice as expert problem solving. Music Perception, v. 20, n. 4, p. 461-485, 2003. http://www.jstor.org/stable/10.1525/mp.2003.20.4.465

CHASE, W. G. (1983). Spatial representation in taxi drivers. In: D. R. ROGERS, J. H. SLOBODA (Eds.), Acquisition of symbolic skills. New York: Plenum, 1983. p. 391-405.

COFFMAN, D.D. Effects of mental practice, physical practice, and knowledge of results in piano performance. Journal of Research in Music Education, v. 38, n. 3, p. 187-196, 1990.

FLAVELL, J.H. Development of children's knowledge about the mental world. International Journal of Behavioral Development, v. 24, n. 1, p. 15-23, 2000. https://doi.org/10.1080/016502500383421 
FIREMAN, M., C. A escolha de repertório na aula de violão como uma proposta cognitiva. Em Pauta, v. 18, n. 30, p. 93-129, 2007. http://www.seer.ufrgs.br/index.php/EmPauta/article/view/7468/4654

GLASER, R.; CHI, M. Overview. In: M. CHI, R. GLASER, M. FARR (Eds.), The nature of expertise. Hillsdale, NJ: Erlbaum, 1998. p. 15-28.

GRAESSER, A.C.; ROBERTSON, S.P.; ANDERSON, P.A. Incorporating Inferences in Narrative Representations: A study of How and Why. Cognitive Psychology, v. 13, n. 1, p. 343-370, 1981.

GOBET, F.; SIMON, H. A. (1996). The roles of recognition processes and look-ahead search in time-constrained expert problem solving: Evidence from grand-master-level chess. Psychological Science, v. 7, n. 1, p. 52-55, 1996. https://doi.org/10.1111/j.1467-9280.1996.tb00666.x

GRANGEAT, M. Les régulations métacognitives dans l'activité enseignante : rôle et modes de développement. Revue des Sciences de l'Éducation, v. 36, n. 1, p. 125-150, 2010. https://doi. org/10.7202/043994ar

GRUHN, W. Are different types of mental representation reflected by brain activation patterns. In: S. D. LIPSCOMB, R. et al. (Eds.), Proceedings of the 8th International Conference on Music perception and Cognition (p. 124-127). Evanston, IL, 2004

. RAUSCHER, F. H. (2008). The neurobiology of learning: New approaches to music pedagogy. In W. GRUHN, F. H. RAUSCHER (Eds.). Neurosciences in music pedagogy. New York: Oxford University Press, 2008. p. 267-282.

JUUTI, S.; LITTLETON, K. (2012). Tracing the Transition from Study to a Contemporary Creative Working Life: The Trajectories of Professional Musicians. Vocations and Learning, v. 5, n. 1, p. 5-21, 2012. https://doi.org/10.1007/s12186-011-9062-9

KERMARREC, G. Stratégies d'apprentissage et autorégulation. Revue de question dans le domaine des habiletés sportives. Science et Motricité, v. 53, n. 3, p. 9-38, 2004. http://dx.doi. org/10.3917/sm.053.0009

KINTSCH, W.; VAN DIJK, T.A. Towards a model of text comprehension and production. Psychological Review, v. 85, n. 5, p. 363-394, 1978. http://dx.doi.org/10.1037/0033-295X.85.5.363

LE BAS, A. Peut-on modéliser les activités physiques en éducation physique et sportive en termes de problématisation? L'exemple de la course de haies. Recherches en Education, v. 11, 164-177, 2001. http://www.recherches-en-education.net/IMG/pdf/REE-no11.pdf

LEHMANN, A. C.; ERICSSON, K. A. Performance without preparation: Structure and acquisition of expert sight-reading and accompanying performance. Psychomusicology, v. 15, n. 1-2, p. 1-29, 1996. http://dx.doi.org/10.1037/h0094082

MARCHAND, C. Pour une didactique de l'art musical. Paris: L'Harmattan, 2009. 312p.

MARCZYK, G.; DeMATTEO, D.; FESTINGER, D. Essentials of Research Designs and Methodology. New Jersey: John Wiley \& Sons, 2005. 306p.

MARTENIUCK, R. G. Information processes in movement learning: capacity and structural interference effects. Journal of Motor Behavior, v. 18, n.1, p. 55-75, 1986. https://doi.org/10.1080/002 22895.1986.10735370

MCPHERSON, G. E.; MCCORMICK, J. Self-efficacy and music performance. Psychology of Music, v. 34, n. 3, p. 321-336, 2006. http://dx.doi.org/ 10.1177/0305735606064841

NIELSEN, S. G. Learning strategies in instrumental music practice. British Journal of Music Education, v. 16, n. 3, p. 275-291, 1999. http://dx.doi.org/10.1017/S0265051799000364 
PHELPS, R.; FERRARA, L.; GOOLSBY, T. W. A Guide to Research in Music Education. 4. ed. New Jersey: The Scarecrow Press, 1993, 367p.

RICHARD, J. F. Les activités mentales. Paris: Armand Colin. 1998. 430p.

ROSENBAUM, D. A. (1987). Hierarchical organization of motor programs. In: S. Wise (Ed.), Neural and behavioral approaches to higher brain functions. New York: Wiley, 1987. p. 45-66.

SCHÖN, D.; AKIVA-KABIRI, L.; VECCHI, T. Psicologia della musica. Roma: Carocci, 2008. 126p.

SEBILLOTTE, S. Décrire des tâches selon les objectifs des opérateurs. De l'interview à la formalisation. Le travail Humain, v. 54, n. 3, p. 193-223, 1991. https://hal.inria.fr/inria-00070042/ document

SWELLER, J. Cognitive load during problem solving: Effects on learning. Cognitive Science. 12, n. 2, p. 257-285, 1998. http://dx.doi.org/10.1207/s15516709cog1202_4

WILLIAMON, A.; VALENTINE, E.; VALENTINE, J. Shifting the focus of attention between levels of musical structure. European Journal of Cognitive Psychology, v. 14, n. 4, p. 493-520, 2002. https://doi.org/10.1080/09541440143000221

WOLF, T. A cognitive model of musical sight-reading. Journal of Psycholinguistic Research, v. 5, n. 2, p. 143-171, 1976.

Michel Cara Jara: Doctor en Ciencias de la Educación, Magister en Psicología Cognitiva Desarrollo y Psicopatología Clínica y Magister en Análisis de Sistemas Educativos, Universidad de Borgoña, Francia. Profesor de Educación Musical, Pontificia Universidad Católica de Chile. Licenciado en Artes con mención en Teoría de la Música, Universidad de Chile. Intérprete con mención en Trombón, Conservatorio Luigi Cherubini, Florencia, Italia. Actualmente es miembro del equipo académico del Instituto de Música PUCV donde imparte las Cátedras de Metodología de la Investigación, Seminario de Título, Práctica Docente Final y Construcción del Pensamiento Infantil Lenguajes Artísticos Integrados. Es igualmente miembro del Laboratorio lenguaje \& Cognición, ELV-PUCV. Sus líneas de investigación son el aprendizaje musical y la formación de profesores. Se especializa en las técnicas de registro de eye-tracking. 